\title{
Negative Differential Conductivity in an Interacting Quantum Gas
}

\author{
Ralf Labouvie, ${ }^{1,2}$ Bodhaditya Santra, ${ }^{1}$ Simon Heun, ${ }^{1}$ Sandro Wimberger, ${ }^{3,4}$ and Herwig $\mathrm{Ott}^{1, *}$ \\ ${ }^{1}$ Research Center OPTIMAS, Technische Universität Kaiserslautern, 67663 Kaiserslautern, Germany \\ ${ }^{2}$ Graduate School Materials Science in Mainz, Staudinger Weg 9, 55128 Mainz, Germany \\ ${ }^{3}$ DiFeST, Università di Parma, Via G. P. Usberti 7/a, 43124 Parma, Italy \\ ${ }^{4}$ INFN, Sezione di Milano Bicocca, Gruppo Collegato di Parma, 43124 Parma, Italy
}

(Received 24 March 2015; published 27 July 2015)

\begin{abstract}
We report on the observation of negative differential conductivity (NDC) in a quantum transport device for neutral atoms employing a multimode tunneling junction. The system is realized with a Bose-Einstein condensate loaded in a one-dimensional optical lattice with high site occupancy. We induce an initial difference in chemical potential at one site by local atom removal. The ensuing transport dynamics are governed by the interplay between the tunneling coupling, the interaction energy, and intrinsic collisions, which turn the coherent coupling into a hopping process. The resulting current-voltage characteristics exhibit NDC, for which we identify atom number-dependent tunneling as a new microscopic mechanism. Our study opens new ways for the future implementation and control of complex neutral atom quantum circuits.
\end{abstract}

Introduction.-Tunneling is a ubiquitous phenomenon exploited in modern electronic transport and detection devices [1-3] as well as in chemical and biological reactions $[4,5]$. Recently, quantum transport phenomena have been observed with ultracold atoms, such as the conductance in mesoscopic channels [6] and its quantization [7], thermoelectric transport [8], and the control of mobility [9]. The conceptual simplicity in such systems allows for a thorough investigation and understanding of the underlying microscopic transport mechanism. Here, we observe negative differential conductivity (NDC) [2] in a multimode tunneling junction for ultracold interacting atoms. Contrary to other prominent implementations in single molecule junctions [10], carbon nanotubes [11], and graphene transistors [12], we demonstrate that NDC originates from a nonlinear, atom number dependent tunneling coupling in combination with fast collisional decoherence. Our many-body device allows for the study of fundamental transport processes influenced by local thermalization or incoherent environments and paves the way for implementations of more complex atomtronic quantum circuits [13-15].

Tunneling transport in solids typically occurs when an electron reservoir with chemical potential (or Fermi energy) $\mu_{1}$ is separated by a tunneling barrier or a quantum well from a second reservoir with chemical potential (or Fermi energy) $\mu_{2}$. This model and extensions of it have been introduced by Esaki and Tsu to calculate the conductance of resonant tunneling diodes and has culminated in the discovery of NDC [2], a phenomenon widely exploited in electronic devices. Here, we give a proof-of-principle for the appearance of NDC in a quantum transport device for neutral atoms residing in an optical lattice. The minimum instance of our experiment is depicted in Fig. 1. Two particle reservoirs with chemical potential $\mu_{1}$ and $\mu_{2}$ are separated by a tunneling barrier and coherently coupled with strength $J$. Each subsystem contains many particles and provides many spatial modes. Preparing an initial nonequilibrium condition, $\Delta \mu=\mu_{1}-\mu_{2} \neq 0$, we measure the ensuing transport through the tunneling barrier. The multimode character of each reservoir is responsible for two distinct physical mechanisms, which govern the tunneling transport: (i) the interaction energy between the particles leads to a density dependent tunneling coupling and (ii) collisions between particles in different modes provide an intrinsic source of decoherence. Both mechanisms together provide the conditions for observing NDC with a steady-state dc current and demarcate the transport dynamics from that of single-mode Josephson junctions [16], where either oscillatory ac currents or pure selftrapping are observed.

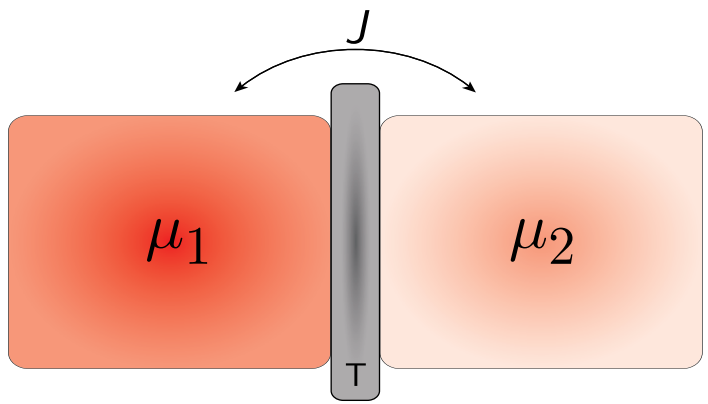

FIG. 1 (color online). System under investigation. Two reservoirs can exchange particles with coupling strength $J$ through a tunneling barrier. The particles in both sides can occupy many spatial modes and have different chemical potentials. 
Experimental system.-We realize this model with a Bose-Einstein condensate of $45 \times 10^{387} \mathrm{Rb}$ atoms in a onedimensional optical lattice with $547 \mathrm{~nm}$ lattice spacing. The trap-frequencies in a lattice site are $\nu_{\rho}=165 \mathrm{~Hz}$ and $\nu_{z}=40-100 \mathrm{kHz}$. Each site contains a two-dimensional Bose-Einstein condensate [17] with $N_{0} \approx 700$ atoms and all atoms reside in the lowest Bloch band. Employing a scanning electron microscopy technique $[18,19]$, we initially remove atoms from one site at the center in a deep lattice where tunneling is absent [the fraction of remaining atoms is about $5 \%-10 \%$ ], thus, creating an imbalance in chemical potential $\Delta \mu$ [Fig. 2(a)]. Lowering the lattice height to different final values, we induce the transport in the array. After variable evolution times, we probe the atom number and the transverse density distribution in the central site using the same electron microscopy technique. Even though the central site is connected to two reservoir sites, collisional decoherence ensures that the experimental system is equivalent to the scheme depicted in Fig. 1.

Figure 2(b) shows the microscopic level structure of the tunneling junction. The chemical potential of the $2 \mathrm{D}$ condensate in a full site is much larger than the level spacing in the radial direction and many spatial modes contribute to the transport. In order to obey energy conservation, the particles can only tunnel into radially excited states of

(a)

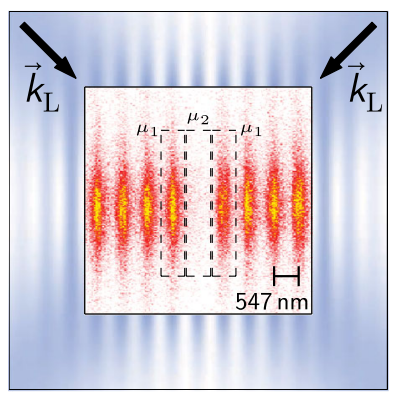

(b)

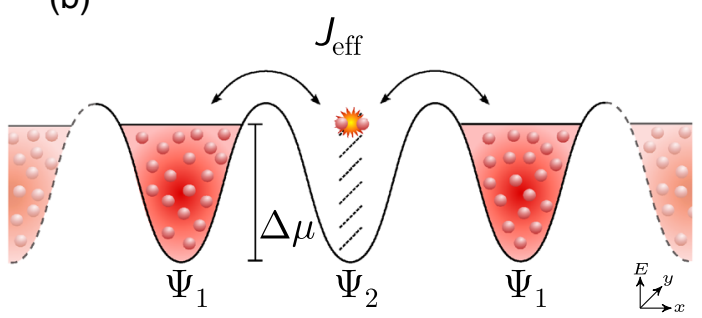

FIG. 2 (color online). (a) Experimental setup: Two blue detuned laser beams $\left(\vec{k}_{\mathrm{L}}\right)$ create a one-dimensional optical lattice in which we load a Bose-Einstein condensate. Removing atoms from the central site of this system leads to an out-of-equilibrium state as an implementation of Fig. 1 (see text). (b) Sketch of the energy level structure (not to scale). Particles of a full site with chemical potential $\mu_{1}$ are resonant with higher radial states of the empty site (indicated as dotted lines) in which they tunnel with a reduced effective tunneling rate $J_{\text {eff }}$. Subsequently, the atoms thermalize by collisional decoherence in the initially empty well. the central site. This goes along with a projection of the radial wave function of the full site onto the available one in the empty site, corresponding to a Franck-Condon factor in the tunneling matrix element. We determine this factor by measuring the radial density distribution of a full site, extracting the modulus of the radial mean-field wave function, $\left|\psi_{1}(\rho)\right|=\sqrt{n(\rho)}$, and calculating the overlap $\eta=\left|\left\langle\psi_{1} \mid \psi_{2}\right\rangle\right|$. Here, $\psi_{2}(\rho)$ is chosen to be the nearest available orbital state of a $2 \mathrm{D}$ harmonic oscillator in the central site that leads to resonant tunneling. This results in an effective tunneling coupling $J_{\text {eff }}(\Delta \mu)$ that depends on the difference in chemical potential between the reservoir and the central site. With increasing $\Delta \mu$, the Franck-Condon factor and, thus, the effective tunneling coupling get smaller. For large $\Delta \mu$, corresponding to an almost empty central site, $J_{\text {eff }}$ is reduced by a factor of 10 with respect to the tunneling coupling $J$ between two full sites, which can be calculated following [20].

Experimental results.-Figure 3(a) shows the experimental results for different values of $J$. In all cases, the
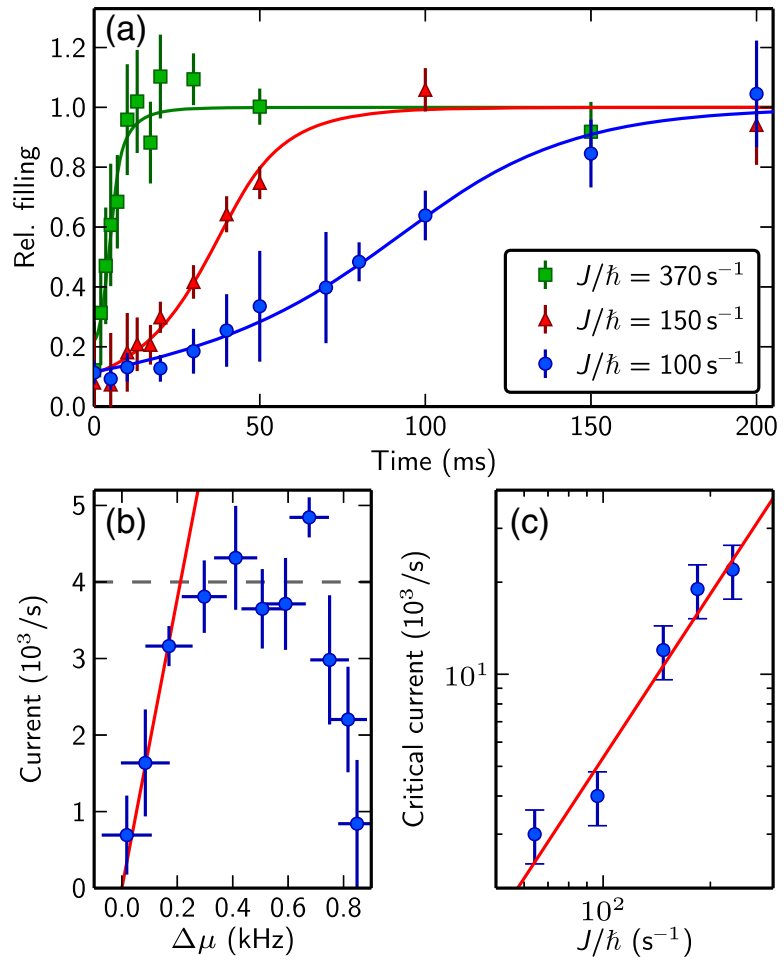

FIG. 3 (color online). Experimental results. (a) Refilling dynamics for three different tunneling couplings. The solid lines are the prediction of our effective model [see Eq. (3) below]. (b) Negative differential conductivity. The experimental points are extracted from the data set with $J / \hbar=100 \mathrm{~s}^{-1}$. After a linear initial rise (red line), the current drops for an increasing imbalance in chemical potential. The dashed line indicates the critical current at which NDC sets in. (c) Critical current for NDC versus coupling strength. Evaluating the critical current for different tunneling couplings $J$, we find a power law $J^{\alpha}$, with $\alpha=1.8(2)$. 
population in the central site smoothly recovers its equilibrium value. The absence of oscillations on top of the refilling curves is a first indicator for the presence of collisional decoherence in the central site. This is in contrast to the Josephson dynamics between two coupled modes [16], where coherent tunneling leads to oscillations of the population. The density dependent tunneling matrix element directly affects the refilling dynamics. For lower values of $J$, the refilling starts visibly with a lower rate and gets faster over time, leading to an overall " $s$-shaped" form.

This behavior can now be converted into a currentvoltage relation: since a difference in chemical potential drives an atomic current through the tunneling junction, it can be identified with an applied voltage. The atomic current is then the equivalent to an electric current. This analogy has been previously applied to characterize the transport of fermionic atoms through mesoscopic channels $[6,7]$. As we will show below, the atomic collisions within the central site guarantee an adiabatic time evolution of the system. Thus, every point of the refilling curve [Fig. 3(a)] represents a steady state and the corresponding tunneling current is obtained by taking the derivative of the refilling curve. The corresponding difference in chemical potential $\Delta \mu$, which represents the applied voltage, is calculated from the population imbalance at a given point [21]. The resulting current-voltage characteristics for the data set with $J / \hbar=100 \mathrm{~s}^{-1}$ are shown in Fig. 3(b). The "s shape" translates into a nonmonotonic dependence. For small $\Delta \mu$, the current increases linearly [see solid line in Fig. 3(b)], which is characteristic for an Ohmic conductivity. But then it bends over, and the current is strongly suppressed for large differences in the chemical potential. These characteristics are known as negative differential conductivity [2,10-12]. It is technically exploited, e.g., in Gunn diodes for the generation of microwave radiation. In a tunnel diode as in most other devices, NDC occurs due to the interplay between the Fermi energy of the carriers on one side of the junction and the density of available hole states on the other side. The latter can be shifted out of resonance or can be depleted by an applied voltage. In superlattice structures, NDC is the result from a competition between localization length and collision rate. Here, however, the microscopic origin of NDC is different: there is neither a detuning nor a change of localization. Instead, the NDC has its origin in a nonlinear tunneling coupling which depends on the applied voltage (or atom number difference). A similar phenomenology is known from correlated hopping [22].

To further characterize this behavior in our system, we extract the critical current $I_{\text {crit }}$ at which NDC sets in for different tunneling couplings $J$ [Fig. 3(c)]. By fitting a power law, we find $I_{\text {crit }} \propto J^{\alpha}$ with $\alpha=1.8(2)$. Tuning the lattice height is, therefore, a knob to adjust the properties of the NDC contact.

The interaction between the atoms also affects the thermodynamic properties of the transport process. Upon tunneling, the interaction energy (given by the chemical potential $\mu_{0}$ of a full site) is converted into kinetic and potential energy, being thermalized after a few collisions. The equilibrium collision rate can be calculated from standard 2D theory [23], and yields values between $150 \mathrm{~s}^{-1}$ and $200 \mathrm{~s}^{-1}$. However, the collision rate for an atom that tunnels into a radially excited state is even higher, as many other atoms initially populate the same state, therefore, being efficiently coupled to neighboring states on a millisecond time scale. Thermal equilibrium is, therefore, rapidly established. The depletion of the condensate in the full site is always balanced by the adjacent reservoir sites and all atoms which tunnel into the central site have the same energy $\mu_{0}$. We can, therefore, apply the equipartition theorem to the 2D harmonic oscillator potential in the central site and find $\mu_{0}=2 k_{\mathrm{B}} T$ for the temperature at the very first part of the dynamics. The prediction of 34(4) $\mathrm{nK}$ is in excellent agreement with our experimental finding 36(2) $\mathrm{nK}$, extracted from a Gaussian fit to the radial density distribution (Fig. 4). This is an extreme manifestation of the Joule Thompson effect in the quantum regime [24], where the interaction energy is converted into thermal energy. For larger filling, the atoms condense, and the width of the density distribution gets smaller. Note that this locally thermalized state occurs even for a global system temperature of $T=0$.

Theoretical model.-The role of decoherence in transport processes has been extensively discussed in the literature $[25,26]$. Its implication for our experiment can be understood from a microscopic description of the tunneling transport. We start from the master equation in Lindblad form [27-31] for the many-body density matrix $\hat{\rho}$

$$
i \hbar \partial_{t} \hat{\rho}=\left[\hat{H}_{\text {eff }}, \hat{\rho}\right]+i \hbar \mathcal{L} \hat{\rho},
$$

where we assume the following dephasing mechanism for the Liouvillian:

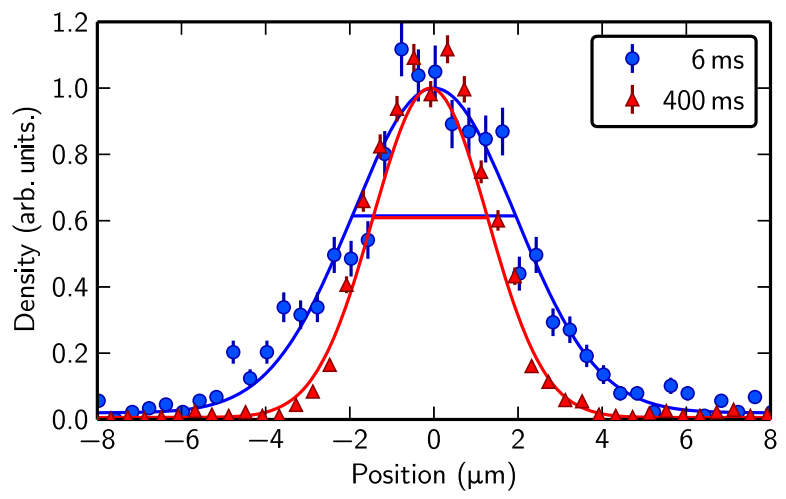

FIG. 4 (color online). Normalized radial density distribution in the central lattice site for the refilling dynamics at $J / \hbar=230 \mathrm{~s}^{-1}$. After $6 \mathrm{~ms}$ (blue dots, 220 atoms), the distribution is governed by thermal energy, and we find a temperature of $T=36(2) \mathrm{nK}$. The equilibrium state (red triangles, 600 atoms), which is reached after $400 \mathrm{~ms}$, is dominated by interaction energy and its width gets smaller. Solid lines are Gaussian fits with horizontal bars indicating their $2 \sigma$ width. 


$$
\mathcal{L} \hat{\rho}=\Gamma_{\operatorname{dec}}\left(2 \hat{n}_{c} \hat{\rho} \hat{n}_{c}^{\dagger}-\hat{n}_{c}^{2} \hat{\rho}-\hat{\rho} \hat{n}_{c}^{2}\right) .
$$

Here, the number operator $\hat{n}_{c}$ acts solely within the central site, where many quantum states in the radial direction are accessible and the atoms can scatter between them. As the available many-body Hilbert space is huge and as the collision rate is much higher than the coherent coupling, we assume thermodynamic equilibrium. It has recently been shown [32] that, in such a situation, the collisional rate in a thermal ensemble of particles is equal to the decoherence rate $\Gamma_{\mathrm{dec}}$.

In principle, we would have to solve the full many-body problem with several radial modes in each lattice site. Since our experimental densities in the initially filled wells are rather large with a filling factor of about 700 , such a microscopic approach would soon become intractable. The large filling, together with the relatively weak two-body scattering for $\mathrm{Rb}$, should guarantee the validity of a mean-field approach, which we are using. Then, the effective Hamiltonian mimics the many-particle behavior of the system with the help of the effective tunnel rate $J_{\text {eff }}(\Delta \mu)$. An exact calculation of the effective tunneling coupling is only possible for a full site and an empty site. In order to describe the behavior of $J_{\text {eff }}(\Delta \mu)$ in a simplified model, we use a linear interpolation between the two values $J$ and $\eta J$, see the definition of the Franck-Condon factor $\eta$ above and [33].

The coupling between all other sites is given by the unchanged tunneling matrix element $J$ since they always have approximately the same filling. Putting all this together, we numerically solve the following equation for the single-particle reduced density matrix $\sigma_{n, m}=$ $\operatorname{tr}\left(\hat{a}_{n}^{\dagger} \hat{a}_{m} \hat{\rho}\right)$ [29-31,34], with the creation and annihilation operators $\hat{a}_{n}^{\dagger}$ and $\hat{a}_{m}$ acting on site $n$ and $m$, respectively,

$$
\begin{aligned}
\dot{\sigma}_{n, m}= & -\frac{i}{\hbar} \operatorname{tr}\left(\hat{a}_{n}^{\dagger} \hat{a}_{m}\left[\hat{H}_{\mathrm{eff}}, \hat{\rho}\right]+\hat{a}_{n}^{\dagger} \hat{a}_{m} \mathcal{L} \hat{\rho}\right) \\
= & \frac{i}{\hbar} J^{\prime}\left(\sigma_{n, m-1}+\sigma_{n, m+1}-\sigma_{n-1, m}-\sigma_{n+1, m}\right) \\
& -\Gamma_{\mathrm{dec}}\left(1-\delta_{n_{c}, m}\right) \sigma_{n_{c}, m}-\Gamma_{\mathrm{dec}}\left(1-\delta_{n, n_{c}}\right) \sigma_{n, n_{c}},
\end{aligned}
$$

where $J^{\prime}=J_{\text {eff }}(\Delta \mu)$ for any index corresponding to the central site $n_{c}$ and $J^{\prime}=J$, otherwise. Without the dephasing term, Eq. (3) would be equivalent to a discrete nonlinear Schrödinger equation for the wave function vector $\left\{\psi_{n}\right\}$ by the identification $\sigma_{n, m}=\psi_{n}^{*} \psi_{m}$ and $\Delta \mu=A N_{0}^{1 / 2} \times$ $\left(\sigma_{n_{c} \pm 1, n_{c} \pm 1}^{1 / 2}-\sigma_{n_{c}, n_{c}}^{1 / 2}\right) \approx A / 2 \cdot \Delta N$, where $A=\left(g m \omega_{\rho}^{2} / \pi\right)^{1 / 2}$. Here, $g$ is the $2 \mathrm{D}$ interaction strength [17], $m$ the mass of the atom, and $\omega_{\rho}$ the radial oscillation frequency. The diagonal element of the single-particle density matrix corresponding to the central site $n_{c}$ is then compared with our experimental data, leaving the decoherence rate $\Gamma_{\text {dec }}$ as a free parameter. The results are shown as solid lines in Fig. 3(a).

Role of decoherence.-This simplified model describes our data very well for all tunneling couplings. We find a
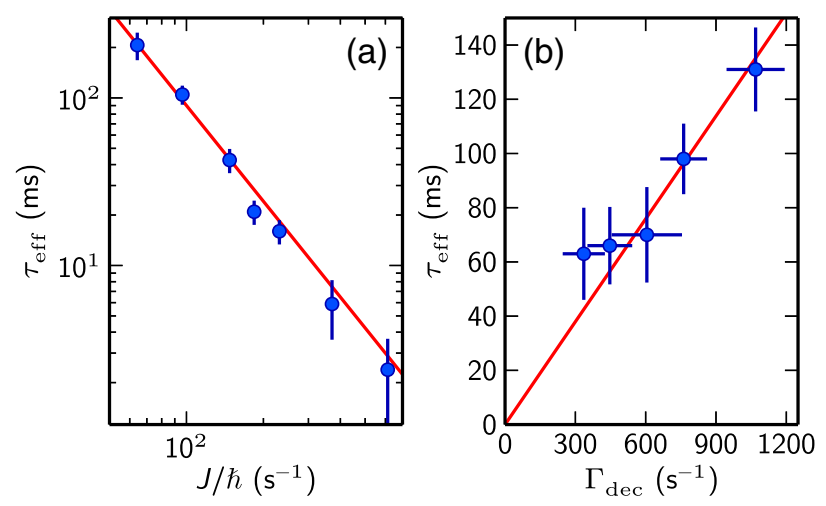

FIG. 5 (color online). Behavior of the time scale for different settings. (a) Refilling time versus tunneling coupling for a constant decoherence rate. We fit a power law with an exponent of $\alpha=1.9(1)$. (b) Refilling time versus decoherence rate: manifestation of the quantum Zeno effect. $\tau_{\text {eff }}$ scales linearly with $\Gamma_{\text {dec }}($ red line).

decoherence rate of about $600 \mathrm{~s}^{-1}$ independent of the tunneling coupling $J$. Its magnitude is in accordance with the above estimated thermal collision rate. We, therefore, identify collisions as the dominant source of decoherence. As the collisions happen much faster than the transport process itself, thermal equilibrium is always established, and indeed, we measure a dc current as if the system was in a steady state at a given time. The decoherence rate is also larger than the tunneling coupling $\left(J / \hbar<400 \mathrm{~s}^{-1}\right)$ and the transport is dominantly incoherent.

The importance of decoherence in our study also manifests itself in the appearance of the quantum Zeno effect. It has been predicted that the effective hopping rate in such a system scales as $\tau_{\text {eff }}^{-1} \propto J^{2} / \Gamma_{\text {dec }}[28,29,35]$. Analyzing the refilling time, which we define as the time constant where the filling exceeds $\sim 67 \%$, in dependence of the tunneling coupling for the constant $\Gamma_{\text {dec }}$ [Fig. 5(a)], we readily recover the quadratic power law: $\tau_{\text {eff }}^{-1} \propto J^{\alpha}$ with $\alpha=1.9(1)$. In order to vary the decoherence rate $\Gamma_{\mathrm{dec}}$, we change the overall atom number, keeping the lattice depth constant $\left(J / \hbar=100 \mathrm{~s}^{-1}\right)$. For $\Gamma_{\mathrm{dec}}>J / \hbar$, we find that $\tau_{\text {eff }} \propto \Gamma_{\text {dec }}$ [Fig. 5(b)]. Given our effective single-particle model, this is in accordance with the seminal results of Caldeira and Leggett [25]. Both findings together verify the predicted dependence: $\tau_{\text {eff }}^{-1} \propto J^{2} / \Gamma_{\text {dec }}$.

Conclusions.-The observation of NDC in the transport of ultracold quantum gases bears great potential for future implementations of interaction controlled atomic circuits [14,15,36-39]. It can also serve as an alternative mechanism for the observation of bistability and related nonequilibrium phenomena. Provided the two reservoirs have different spatial geometries, the transport process might become even asymmetric as a function of $\Delta \mu$. Such an asymmetry translates into a left-right transport imbalance and might provide a possible strategy to build a diode for neutral matter currents. On a more fundamental level, our 
approach to nonequilibrium dynamics, following a quench in the density, allows for an in-depth study and understanding of local thermalization processes and many-body transport phenomena in incoherent environments.

We acknowledge discussions with James Anglin, Ennio Arimondo, Georgios Kordas, Artur Widera, and Dirk Witthaut, and financial support by the DFG within the SFB/TRR 49 and WI 3426/7. R. L. is supported by the MAINZ graduate school.

*tt@physik.uni-kl.de

[1] R. C. Jaklevic, J. Lambe, A. H. Silver, and J. E. Mercereau, Phys. Rev. Lett. 12, 159 (1964).

[2] L. Esaki and R. Tsu, IBM J. Res. Dev. 14, 61 (1970).

[3] S. H. Choi, B. Kim, and C. D. Frisbie, Science 320, 1482 (2008).

[4] J. Winkler, H. Gray, T. Prytkova, I. Kurnikov, and D. Beratan, Bioelectronics: From Theory to Applications (Wiley-VCH, Weinheim, 2005), pp. 15-33.

[5] Y. Cha, J. M. Murray, and J. P. Klinman, Science 243, 1325 (1989).

[6] J.-P. Brantut, J. Meinike, D. Stadler, S. Krinner, and T. Esslinger, Science 337, 1069 (2012).

[7] S. Krinner, D. Stadler, D. Husmann, J.-P. Brantut, and T. Esslinger, Nature (London) 517, 64 (2015).

[8] E. L. Hazlett, L.-C. Ha, and C. Chin, arXiv:1306.4018.

[9] T. Salger, S. Kling, S. Denisov, A. V. Ponomarev, P. Hänggi, and M. Weitz, Phys. Rev. Lett. 110, 135302 (2013).

[10] M. L. Perrin, C. J. O. Verzijl, C. A. Martin, A. J. Shaikh, R. Eelkema, J. H. van Esch, J. M. van Ruitenbeek, J. M. Thijssen, H. S. J. van der Zant, and D. Dulić, Nat. Nanotechnol. 8, 282 (2013).

[11] E. Pop, D. Mann, J. Cao, Q. Wang, K. Goodson, and H. Dai, Phys. Rev. Lett. 95, 155505 (2005).

[12] L. Britnell, R. V. Gorbachev, A. K. Geim, L. A. Ponomarenko, A. Mishchenko, M. T. Greenaway, T. M. Fromhold, K. S. Novoselov, and L. Eaves, Nat. Commun. 4, 1794 (2013).

[13] A. Micheli, A. J. Daley, D. Jaksch, and P. Zoller, Phys. Rev. Lett. 93, 140408 (2004).

[14] R. A. Pepino, J. Cooper, D. Z. Anderson, and M. J. Holland, Phys. Rev. Lett. 103, 140405 (2009).

[15] S. Eckel, J. G. Lee, F. Jendrzejewski, N. Murray, C. W. Clark, C. J. Lobb, W. D. Phillips, M. Edwards, and G. K. Campbell, Nature (London) 506, 200 (2014).

[16] M. Albiez, R. Gati, J. Fölling, S. Hunsmann, M. Cristiani, and M. K. Oberthaler, Phys. Rev. Lett. 95, 010402 (2005).
[17] Z. Hadzibabic and J. Dalibard, Rivista del Nuovo Cimento 34, 389 (2011).

[18] T. Gericke, P. Würtz, D. Reitz, T. Langen, and H. Ott, Nat. Phys. 4, 949 (2008).

[19] G. Barontini, R. Labouvie, F. Stubenrauch, A. Vogler, V. Guarrera, and H. Ott, Phys. Rev. Lett. 110, 035302 (2013).

[20] I. Bloch, J. Dalibard, and W. Zwerger, Rev. Mod. Phys. 80, 885 (2008).

[21] See Supplemental Material at http://link.aps.org/ supplemental/10.1103/PhysRevLett.115.050601 for more details on how to extract $\Delta \mu$.

[22] I. N. Karnaukhov, Phys. Rev. Lett. 73, 1130 (1994).

[23] D. Guéry-Odelin and T. Lahaye, C.R. Phys. 5, 55 (2004).

[24] T. F. Schmidutz, I. Gotlibovych, A. L. Gaunt, R. P. Smith, N. Navon, and Z. Hadzibabic, Phys. Rev. Lett. 112, 040403 (2014).

[25] A. O. Caldeira and A. J. Leggett, Ann. Phys. (N.Y.) 149, 374 (1983).

[26] Y. Imry and R. Landauer, Rev. Mod. Phys. 71, S306 (1999).

[27] H.-P. Breuer and F. Petruccione, The Theory of Open Quantum Systems (Oxford University Press, Oxford, 2002).

[28] P. Barmettler and C. Kollath, Phys. Rev A 84, 041606(R) (2011).

[29] F. Trimborn, D. Witthaut, H. Hennig, G. Kordas, T. Geisel, and S. Wimberger, Eur. Phys. J. D 63, 63 (2011).

[30] D. Witthaut, F. Trimborn, H. Hennig, G. Kordas, T. Geisel, and S. Wimberger, Phys. Rev. A 83, 063608 (2011).

[31] G. Kordas, S. Wimberger, and D. Witthaut, Europhys. Lett. 100, 30007 (2012).

[32] L. Gilz, L. Rico-Perez, and J. R. Anglin, Phys. Rev. A 89, 052131 (2014).

[33] See Supplemental Material at http://link.aps.org/ supplemental/10.1103/PhysRevLett.115.050601 for more details on the conversion from atom number into chemical potential.

[34] G. Kordas, S. Wimberger, and D. Witthaut, Phys. Rev. A 87, 043618 (2013).

[35] A. G. Kofman and G. Kurizki, Nature (London) 405, 546 (2000).

[36] P. Schlagheck, F. Malet, J. C. Cremon, and S. M. Reimann, New J. Phys. 12, 065020 (2010).

[37] S. C. Caliga, C. Straatsma, A. A. Zozulya, and D. Z. Anderson, arXiv:1208.3109.

[38] D. B. Gutman, Y. Gefen, and A. D. Mirlin, Phys. Rev. B 85, 125102 (2012).

[39] A. Ivanov, G. Kordas, A. Komnik, and S. Wimberger, Eur. Phys. J. B 86, 345 (2013). 